\title{
Breast cancer: a new imaging approach as an addition to existing guidelines
}

\author{
Monique D. Dorrius • Erik F. J. de Vries • \\ Riemer H. J. A. Slart • Andor W. J. M. Glaudemans
}

Received: 29 January 2015 / Accepted: 5 February 2015 /Published online: 12 March 2015

(C) Springer-Verlag Berlin Heidelberg 2015

\section{Introduction}

Worldwide breast cancer has the highest incidence of all malignancies in women. Although the incidence has increased, mortality has decreased during the last two decades [1]. This reduction in mortality is largely due to early detection of malignancies in screening and to better and more personalized therapies [2].

Mammography is the primary screening imaging modality. This technique, however, has limitations in terms of sensitivity (39-86\%) and specificity (88-94\%), depending on age and breast density [3, 4]. Therefore, ultrasound is used in addition to mammography to increase diagnostic accuracy [5]. Mammography is scored in five categories according to the American College of Radiology (ACR) Breast Imaging Reporting and Data System (BI-RADS): category 1, negative; 2, benign finding; 3, probably benign; 4, suspicious finding (chance of malignancy 2-95\%); and 5, highly suggestive of malignancy (chance of malignancy $>95 \%$ ) [6]. The guideline for non-invasive diagnostic tests for breast abnormalities of the Agency for Healthcare Research and Quality (AHRQ) in the USA states that for BI-RADS 1 and 2 no further work-up is required. The work-up for BI-RADS 4 and 5 requires an

\footnotetext{
M. D. Dorrius

Department of Radiology, University of Groningen, University

Medical Center Groningen, Groningen, The Netherlands

E. F. J. de Vries · R. H. J. A. Slart • A. W. J. M. Glaudemans ( $\square)$ Department of Nuclear Medicine and Molecular Imaging, University of Groningen, University Medical Center Groningen, Hanzeplein 1, PO Box 30.001, 9700 RB Groningen, The Netherlands e-mail: a.w.j.m.glaudemans@umcg.nl
}

R. H. J. A. Slart

Biomedical Photonic Imaging Group, University of Twente, Enschede, The Netherlands invasive procedure (biopsy). The diagnostic work-up of a BIRADS 3 lesion (chance of malignancy $<2 \%$ ) can be either biopsy or follow-up mammography after 6 months; in the recent 5 th edition of the ACR guideline only a short interval-follow-up (6 month) or continue surveillance is recommended $[6,7]$. However, in daily practice most of the patients with a non-calcified BI-RADS 3 and/or 4 lesion are referred for biopsy for histological confirmation. Approximately $95 \%$ of the patients with a non-calcified BI-RADS 3 and $80 \%$ of the patients with a non-calcified BI-RADS 4 lesion eventually will have a benign lesion [6, 8]. Diagnostic imaging techniques able to exclude malignancy with a high level of evidence, allowing avoidance of unnecessary invasive biopsy procedures, would be of major benefit.

In proven breast cancer, National Comprehensive Cancer Network (NCCN) and European Society for Medical Oncology (ESMO) clinical practice guidelines indicate that additional imaging techniques could play a role in breast cancer stage II or higher in order to confirm the presence of locoregional or distant metastases. Assessment of dissemination of the disease can be considered for stage $\geq$ II breast cancer based on clinically positive axillary nodes, large tumours (e.g. $>5 \mathrm{~cm}$ ), clinical symptoms or laboratory values suggesting the presence of metastases $[9,10]$. In the case of proven breast cancer, imaging modalities can have an added value not only to determine the extent of the disease but also to characterize the primary and metastatic breast lesions.

Non-invasive modalities are increasingly implemented in standard care to support breast cancer diagnosis, staging and evaluation. However, the role of several potential imaging investigations has not yet been fully established and existing international guidelines are lagging behind these developments. The purpose of this editorial is to provide guidance for the application of (developing) imaging techniques in (1) the non-invasive work-up of breast lesions and (2) staging and characterization of advanced breast cancer. 


\section{Diagnostic imaging in patients with non-calcified mammographic BI-RADS 3 and 4 lesions}

Better application of non-invasive imaging techniques may significantly reduce the number of unnecessary invasive biopsy procedures in patients with non-calcified mammographic BI-RADS 3 and 4 breast lesions, as is emphasized in the left part of the flow chart (Fig. 1).

In cases of equivocal non-calcified mammographic BIRADS 3 and 4 lesions, dynamic contrast-enhanced (DCE) MRI should be the first choice non-invasive imaging modality. DCE-MRI has the highest overall negative predictive value (NPV) of all imaging techniques and is able to safely exclude malignancy (NPV>98\%) [8, 11-14]. Only in cases with pure clustered microcalcifications is the NPV lower [8, 15]. However, DCE-MRI is not implemented in the guidelines for diagnosis of non-calcified mammographic BI-RADS 3 and 4 lesions yet, as few data are available to support the use of DCE-MRI as a problem solving modality. However, this modality might soon deserve a place in the guidelines, as studies showed that in about $68-92 \%$ of the patients with a non-calcified mammographic BI-RADS 3 and 4 lesion DCEMRI can avoid a diagnostic invasive biopsy $[8,11]$. In the remaining breast lesions (8-32\%), there is enhancement of DCE-MRI, but with considerable overlap in enhancement patterns of benign and malignant breast lesions. Therefore, the majority of BI-RADS 3 and 4 lesions showing enhancement on DCE-MRI are considered undecided. The specificity of
DCE-MRI might well increase in the future, if promising sequences like diffusion-weighted imaging (DWI) and multivoxel MR spectroscopy (MRS) are added to the standard breast MRI protocol. The estimation is that an unnecessary biopsy can be avoided in approximately $50 \%$ of the cases with enhanced lesions on DCE-MRI, if DWI and/or MRS is added $[11,16]$.

DWI has a higher specificity (75-84\%) [17] than DCEMRI (67-72\%) [18]. DWI is an MRI method that reflects the diffusion of water molecules through biological tissues. The DWI signal attenuation can be quantified as the apparent diffusion coefficient (ADC) [19]. It is important to realize that there are no standard measurement protocols and methods of data analysis for DWI and consequently incorrect procedures can lead to false-negative and false-positive results. The ADC values are affected by acquisition parameters like the strength and duration of diffusion gradients as well as by physiological parameters like micro-perfusion [17]. Therefore, DWI should be quantified using a bi-exponential model like the intra-voxel incoherent motion (IVIM) model, resulting in increased diagnostic performance as compared to ADC [20].

In vivo proton MRS of the breast provides metabolic information about e.g. choline levels in the investigated tissue in a non-invasive manner. Although MRS is a more complicated sequence than DWI, its short sequence (ca. $10 \mathrm{~min}$ ) should also be added to the standard breast MRI protocol, because the accuracy of multi-voxel MRS added to the breast MRI BIRADS classification exceeds the accuracy of MRI alone

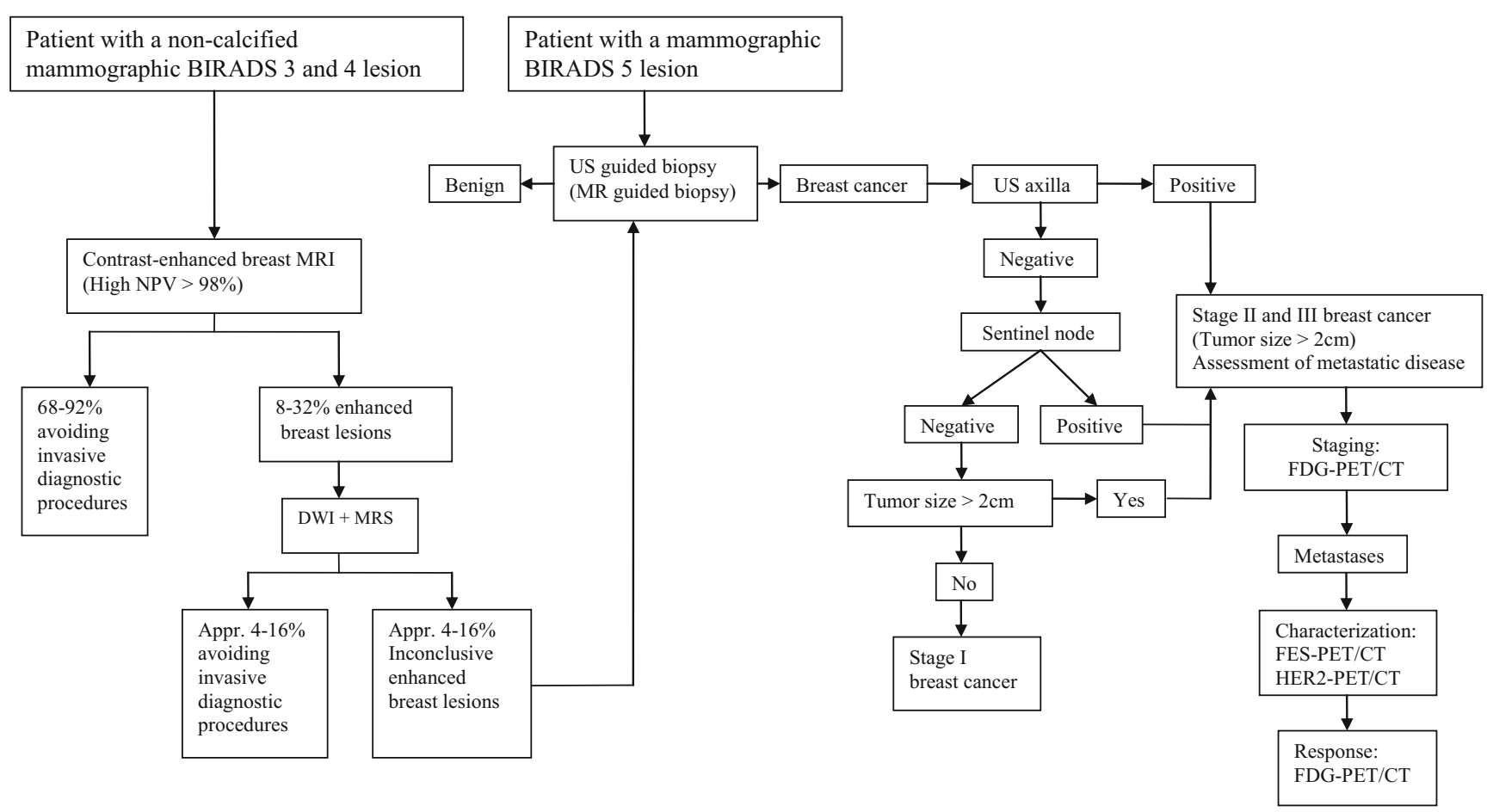

Fig. 1 Flow chart 
[16]. Preferably, a multi-voxel method should be used, instead of a single-voxel method, to quantify choline levels. A choline signal is not only detected in tumour lesions, but also in benign breast lesions and normal fibroglandular tissue, and consequently accurate quantification is required to discriminate benign from malignant lesions [21].

In a diagnostic algorithm of non-calcified mammographic BI-RADS 3 and 4 lesions, the combination of DCE-MRI, DWI and multi-voxel MRS is the most optimal non-invasive work-up to avoid unnecessary invasive procedures. A study in 113 patients showed that the NPV of this approach was $100 \%$ [22], provided that lesions are larger than $1 \mathrm{~cm}$ in order to avoid partial volume effects in DWI and MRS. However, it is expected that smaller lesions can also be analysed with 3.0-T MRI scanners, which have a higher spatial resolution.

Although DCE-MRI is the modality of choice for the diagnosis of non-calcified mammographic BI-RADS 3 and 4 lesions, positron emission tomography/computed tomography (PET/CT) or positron emission mammography (PEM) with ${ }^{18}$ F-fluorodeoxyglucose (FDG) could possibly play a role when MRI is contraindicated (e.g. claustrophobia, metal implants). In a study with 76 patients the sensitivity $(96 \%)$ and specificity (70\%) of FDG PET are comparable with those of DCE-MRI [23]. However, also FDG PET has its limitations. False-negative findings on FDG PET/CT are reported in small lesions or slowly proliferating (non-FDG-avid) breast lesions [24]. Falsepositive FDG uptake can occur in inflammatory or infectious lesions. Moreover, this technique is expensive.

The added value of PEM is still under debate. PEM was introduced as having the advantage of a better spatial resolution $(2.4 \mathrm{~mm})$ than conventional PET/CT $[25,26]$ and therefore it should be able to detect smaller lesions. However, modern PET/CT cameras can also obtain reconstructed resolutions that are in the same range and therefore this advantage has largely disappeared. In a meta-analysis, PEM has a pooled sensitivity and specificity of 84 and $79 \%$, respectively [27] and thus cannot outperform FDG PET/CT or DCE-MRI.

In general, clinicians have to think wisely about which imaging tests should be recommended, especially if the aim is to exclude cancer. Not only diagnostic performance, but also the risks, such as exposing the patient to radiation, and costs should be considered. The radiation dose of FDG PET/ CT is approximately $4.0 \mathrm{mSv}$ for an adult person of $70 \mathrm{~kg}$ when the correct acquisition protocols are applied and therefore comparable with the radiation burden of diagnostic CT scans. This radiation dose should not be considered as an absolute contraindication anymore. However, MRI remains favourable since this imaging technique does not expose patients to radiation and has the highest NPV of all imaging techniques. Therefore, MRI is the best technique to avoid unnecessary invasive procedures.
Diagnostic imaging in patients with proven breast cancer

When a suspected breast lesion is detected on the mammogram or DCE-MRI, the guideline states that patient will undergo a biopsy procedure and an ultrasound of the axilla to screen for suspicious lymph nodes. When breast cancer is proven by biopsy and the ultrasound of the axilla is negative, a sentinel node procedure will be performed. As mentioned in the "Introduction", additional imaging techniques could play a role in breast cancer stage II or higher to determine whether locoregional or distant metastases are present $[9,10]$ (Fig 1; right part of the flow chart).

To determine if the patient has distant metastases, nowadays the patient will undergo chest radiography/liver ultrasound or a CT scan (chest and/or abdominal) and bone scintigraphy in routine clinical practice. FDG PET is superior to conventional imaging procedures (chest X-ray, abdominal ultrasound and bone scintigraphy) for detection of distant metastases. The overall sensitivity and specificity of FDG PET in detecting distant metastases is 100 and $98 \%$, respectively, whereas the sensitivity and specificity of conventional imaging is 60 and $83 \%$, respectively [28]. Furthermore, FDG PET identifies bone metastases with higher accuracy than bone scintigraphy [29]. The diagnostic accuracy of FDG PET is comparable with contrast-enhanced CT scan. FDG PET has clinically relevant advantages in the detection of lymph node metastases, particularly if these lymph nodes have a normal diameter. The contrast-enhanced CT scan has distinct advantages in the identification of both small lung and liver metastases [29]. Therefore, in our opinion, for dissemination purposes conventional imaging should be replaced by a combination of FDG PET and contrast-enhanced CT scan. Furthermore, there is a high possibility that PET/CT will extensively be used in the future for treatment decision-making in breast cancer and for monitoring the response to therapy. Therefore, a baseline FDG PET/CT scan may be recommended in future guidelines.

\section{Future developments}

Nowadays, the assessment of therapy response is based on the RECIST1.1 criteria that are validated and largely obtained with CT scanning. Regretfully, most bone metastases are often not evaluable with RECIST1.1. Furthermore, therapy response with FDG PET has not yet been validated. At the moment, the use of FDG PET/CT for determination of early treatment response is being assessed (IMPACT-MBC trial, NCT01957332).

In this trial, also molecular characteristics of the primary tumour and metastases are assessed that are relevant for targeted therapy (personalized medicine). Oestrogen receptors (ER) are over-expressed in around $70 \%$ of all breast cancers 
and are a target for endocrine therapy [30]. Human epidermal growth factor receptor 2 (HER2) is over-expressed in approximately $25 \%$ of all breast cancers and is a target for therapy such as trastuzumab, a monoclonal antibody against the HER2 [30]. ER and HER2 expression can change over time, and in up to $40 \%$ of the patients discordant expression is seen between primary tumour and metastases [31]. Therefore, currently when patients develop metastatic disease rebiopsy is advised. Whole-body non-invasive PET imaging with $16 \alpha-{ }^{18} \mathrm{~F}$-fluoro- $17 \beta$-oestradiol $\left({ }^{18} \mathrm{~F}\right.$-FES) and ${ }^{89} \mathrm{Zr}$ trastuzumab enables visualization and quantification of all tumour lesions within a patient [32]. These novel and more specific techniques may be used to improve personalized treatment decisions for the individual patient and might improve prediction of response to therapy. This hypothesis is at this moment being evaluated in a multicentre trial in which patients with metastatic breast cancer eligible for first-line systemic therapy undergo ${ }^{18} \mathrm{~F}$-FES PET/CT and ${ }^{89} \mathrm{Zr}$ trastuzumab PET/CT (IMPACT-MBC trial, NCT01957332).

Another interesting development in hybrid imaging is the simultaneous PET/MRI camera. As this technique is still in its infancy, the added value of PET/MRI is still questionable. At this moment, there is no indication that FDG PET/MRI could have any added clinical value compared to FDG PET and MRI alone.

\section{Conclusion}

This editorial provides guidance for the application of (developing) non-invasive imaging techniques that can be implemented in patients with suspected breast lesions. Some techniques could be implemented right away in already existing guidelines, while other techniques still have to be carefully evaluated for their added value. Breast MRI can be used to safely rule out malignancy in non-calcified breast lesions when using the optimal sequences (DCE, DWI and multi-voxel MRS) and the correct analysis techniques. FDG $\mathrm{PET} / \mathrm{CT}$ could replace the conventional imaging techniques in disseminated disease. In the near future, an important role is to be expected for PET techniques that are able to characterize the tumour and its metastases, thereby making a selection of individual treatment options and assessment of the response to therapy possible.

\section{References}

1. Paap E, Broeders MJ, van Schoor G, Otten JD, Verbeek AL. Large increase in a Dutch woman's lifetime risk of developing breast cancer. Eur J Cancer 2008;44:1485-7.

2. Berry DA, Cronin KA, Plevritis SK, Fryback DG, Clarke L, Zelen M, et al. Effect of screening and adjuvant therapy on mortality from breast cancer. N Engl J Med 2005;353:1784-92.
3. Barlow WE, Lehman CD, Zheng Y, Ballard-Barbash R, Yankaskas $\mathrm{BC}$, Cutter GR, et al. Performance of diagnostic mammography for women with signs or symptoms of breast cancer. J Natl Cancer Inst 2002;94:1151-9.

4. Carney PA, Miglioretti DL, Yankaskas BC, Kerlikowske K, Rosenberg R, Rutter CM, et al. Individual and combined effects of age, breast density, and hormone replacement therapy use on the accuracy of screening mammography. Ann Intern Med 2003;138: $168-75$.

5. Zonderland HM, Coerkamp EG, Hermans J, van de Vijver MJ, van Voorthuisen AE. Diagnosis of breast cancer: contribution of US as an adjunct to mammography. Radiology 1999;213:413-22.

6. American College of Radiology (ACR). Illustrated breast imaging reporting and data system (BI-RADS). Mammography, ultrasound and MRI. 5th ed. 2013.

7. Agency for Health Care Research and Quality. Effectiveness of noninvasive diagnostic test for breast abnormalities. Accessed AHRQ publication no. 06-EHC005-EF. 2009.

8. Strobel K, Schrading S, Hansen NL, Barabasch A, Kuhl CK. Assessment of BI-RADS category 4 lesions detected with screening mammography and screening US: utility of MR imaging. Radiology 2015;274:343-51.

9. National Comprehensive Cancer Network (NCCN). Evidence-based cancer guidelines. http://www.ncen.org/. 2015.

10. European Society for Medical Oncology (ESMO). The clinical practice guidelines. http://www.esmo.org/. 2015.

11. Dorrius MD, Pijnappel RM, Sijens PE, van der Weide MC, Oudkerk $\mathrm{M}$. The negative predictive value of breast magnetic resonance imaging in noncalcified BIRADS 3 lesions. Eur J Radiol 2012;81:20913.

12. Gokalp G, Topal U. MR imaging in probably benign lesions (BI-RADS category 3) of the breast. Eur J Radiol 2006;57: 436-44.

13. Mahoney MC, Gatsonis C, Hanna L, DeMartini WB, Lehman C. Positive predictive value of BI-RADS MR imaging. Radiology 2012;264:51-8.

14. Moy L, Elias K, Patel V, Lee J, Babb JS, Toth HK, et al. Is breast MRI helpful in the evaluation of inconclusive mammographic findings? AJR Am J Roentgenol 2009;193:986-93.

15. Bluemke DA, Gatsonis CA, Chen MH, DeAngelis GA, DeBruhl N, Harms S, et al. Magnetic resonance imaging of the breast prior to biopsy. JAMA 2004;292:2735-42.

16. Dorrius MD, Pijnappel RM, van der Weide Jansen MC, Jansen L, Kappert P, Oudkerk M, et al. The added value of quantitative multivoxel MR spectroscopy in breast magnetic resonance imaging. Eur Radiol 2012;22:915-22.

17. Dorrius MD, Dijkstra H, Oudkerk M, Sijens PE. Effect of b value and pre-admission of contrast on diagnostic accuracy of 1.5-T breast DWI: a systematic review and meta-analysis. Eur Radiol 2014;24: 2835-47.

18. Peters NH, Borel Rinkes IH, Zuithoff NP, Mali WP, Moons KG, Peeters PH. Meta-analysis of MR imaging in the diagnosis of breast lesions. Radiology 2008;246:116-24.

19. Le Bihan D, Breton E, Lallemand D, Aubin ML, Vignaud J, LavalJeantet M. Separation of diffusion and perfusion in intravoxel incoherent motion MR imaging. Radiology 1988;168:497-505.

20. Liu C, Liang C, Liu Z, Zhang S, Huang B. Intravoxel incoherent motion (IVIM) in evaluation of breast lesions: comparison with conventional DWI. Eur J Radiol 2013;82:e782-9.

21. Dorrius MD, Pijnappel RM, Jansen-van der Weide MC, Jansen L, Kappert P, Oudkerk M, et al. Determination of choline concentration in breast lesions: quantitative multivoxel proton MR spectroscopy as a promising noninvasive assessment tool to exclude benign lesions. Radiology 2011;259:695-703.

22. Pinker K, Bogner W, Baltzer P, Gruber S, Bickel H, Brueck B, et al. Improved diagnostic accuracy with multiparametric magnetic 
resonance imaging of the breast using dynamic contrast-enhanced magnetic resonance imaging, diffusion-weighted imaging, and 3dimensional proton magnetic resonance spectroscopic imaging. Invest Radiol 2014;49:421-30.

23. Pinker K, Bogner W, Baltzer P, Karanikas G, Magometschnigg H, Brader $\mathrm{P}$, et al. Improved differentiation of benign and malignant breast tumors with multiparametric 18fluorodeoxyglucose positron emission tomography magnetic resonance imaging: a feasibility study. Clin Cancer Res 2014;20:3540-9.

24. Escalona S, Blasco JA, Reza MM, Andradas E, Gómez N. A systematic review of FDG-PET in breast cancer. Med Oncol 2010;27:114 29.

25. Kalinyak JE, Berg WA, Schilling K, Madsen KS, Narayanan D, Tartar M. Breast cancer detection using high-resolution breast PET compared to whole-body PET or PET/CT. Eur J Nucl Med Mol Imaging 2014;41:260-75.

26. MacDonald L, Edwards J, Lewellen T, Haseley D, Rogers J, Kinahan P. Clinical imaging characteristics of the positron emission mammography camera: PEM Flex Solo II. J Nucl Med 2009;50:1666-75.

27. Caldarella C, Treglia G, Giordano A. Diagnostic performance of dedicated positron emission mammography using fluorine-18- fluorodeoxyglucose in women with suspicious breast lesions: a meta-analysis. Clin Breast Cancer 2014;14:241-8.

28. Fuster D, Duch J, Paredes P, Velasco M, Muñoz M, Santamaría G, et al. Preoperative staging of large primary breast cancer with $[18 \mathrm{~F}]$ fluorodeoxyglucose positron emission tomography/computed tomography compared with conventional imaging procedures. J Clin Oncol 2008;26:4746-51.

29. Mahner S, Schirrmacher S, Brenner W, Jenicke L, Habermann CR, Avril N, et al. Comparison between positron emission tomography using 2-[fluorine-18]fluoro-2-deoxy-D-glucose, conventional imaging and computed tomography for staging of breast cancer. Ann Oncol 2008;19:1249-54.

30. Early Breast Cancer Trialists' Collaborative Group (EBCTCG). Effects of chemotherapy and hormonal therapy for early breast cancer on recurrence and 15-year survival: an overview of the randomised trials. Lancet 2005;365:1687-717.

31. van Kruchten M, de Vries EG, Brown M, de Vries EF, Glaudemans AW, Dierckx RA, et al. PET imaging of oestrogen receptors in patients with breast cancer. Lancet Oncol 2013;14:e465-75.

32. Oude Munnink TH, Nagengast WB, Brouwers AH, Schröder CP, Hospers GA, Lub-de Hooge MN, et al. Molecular imaging of breast cancer. Breast 2009;18 Suppl 3:S66-73. 